\title{
Grand Challenges in loT and Sensor Networks
}

\author{
Muhammad Ali Imran*, Ahmed Zoha, Lei Zhang and Qammer H. Abbasi \\ James Watt School of Engineering, University of Glasgow, Glasgow, United Kingdom
}

Keywords: internet of things (IOT), sensor networks (SN), artificial intelligence, wireless communication, privacy and security, verticals of IoT

\section{INTRODUCTION}

The exponential growth in mobile traffic seen in the last couple of years, mainly due to the vast amount of wireless devices, such as smart phones and the Internet of Things (IoT), has resulted in the wireless network industry producing and collecting an unprecedented amount of data (Bi et al., 2015). According to the International Data Corporation, by 2025 the number of devices connected to the Internet will be around 42 billion, and a total of 80 zettabytes of data will be generated in the same year. There is no doubt that we are ushering in a new era, since IoT and Artificial Intelligence (AI) are deepening their integration in society and the roll-out of 5G technology will spur new innovations across all industries.

The innovation in the IoT ecosystem is bridging the gap between the real and digital world; we are creating a hyper-connected society where devices are no longer used only to exchange data but are becoming more and more intelligent and context aware. The advancements in sensing, data processing, and cloud and communication technology has enabled the systems to interact with the environment and optimize processes via learning through interactions. This will lead to the creation of smart spaces and self-aware interconnected "things" for health, mobility, digital society, food, energy, and environmental applications (Qiu et al., 2018).

In future, the IoT development process will most likely evolve from vertical to polymorphic applications, supporting both personal and industry users (Chen et al., 2014). To provide a pervasive, unified, and seamless experience to the end users, there are many challenges that need to be addressed, including: technology standards, interoperable module components supporting heterogeneous applications and requirements at several layers, the designing of low-cost IoT terminals with low-active power, and solutions guaranteeing end-to-end privacy and security (Chen et al., 2014). Several grand challenges inherent to IoT systems are outlined in the following sections.

\section{GRAND CHALLENGES IN IOT}

\section{Scalability}

Given the huge number of devices requiring simultaneous connectivity, scalability in IoT systems has become a concern. In IoT, there are mainly two types of scalability issues: vertical scalability, which refers to the addition or removal of computing resources of an IoT node; and horizontal scalability, which refers to the addition or removal of an IoT node. Given its importance, IoT scalability has been extensively addressed in the literature, with the proposal of cloud computing (Cabré et al., 2017; Cheng et al., 2017; Shirer and MacGillivray, 2020) or cloud-based architectures (Xu and Helal, 2015). However, despite these efforts, challenges still remain, such as IoT nodes needing to provide an increased number of services, such as functional scalability, access control, data storage, fault tolerance, and privacy and security, to name a few. 


\section{Security and Privacy}

The lack of privacy standards and end-to-end security solutions has been an ongoing concern for conventional IoT deployment, and wireless IoT faces more challenges in terms of these aspects (Shafagh et al., 2017). Several technologies are aiming to solve privacy and security issues from both a hardware and software perspective. For hardware, RFID and newer releases of $5 \mathrm{G}$ and other local network protocol are key to tackling security issues at a hardware level. In terms of software, Key Management System (KMS) with a zero-trust network feature and blockchain are rapidly addressing the privacy and trust threats with reinforced security features (Sicari et al., 2015; Sun et al., 2019; Xu et al., 2020). With the help of newer communication protocols, KMS, and blockchain, the grand challenge of IoT devices is the interdependency of security, privacy, and trust for IoT ecosystems. The challenge shall always be considered as a holistic goal for optimal integrity and performance.

\section{Self-Organization}

There is an ongoing paradigm shift from Internet-of-Things to Internet-of-Everything due to the proliferation of IoT nodes which demands new approaches to autonomic management to make the network proactive rather than reactive. The main idea behind self-organization in IoT systems is to actively respond to the changing environments in an automatic and coordinated fashion through the use of one or more control loops that reconfigures the system behavior on-demand to keep it within desired bounds (Kephart, 2005). These systems proactively selfmanage themselves by reacting to the changing environment using advanced algorithms in conjunction with high-level human-defined goals and policies. These self-organization capabilities are important to ensure the robustness and survival of the future dense IoT network and therefore have attracted an intense research interest (Milner et al., 2012; Ding et al., 2013; Qiu et al., 2017; Pang et al., 2020). However, there are many open challenges in this space and some notable research directions for the future include dealing with the heterogenous interoperability of the system, designing of optimal self-organizing protocols and routing strategies for large-scale distributed heterogenous IoT networks, and cross-platform behavior optimization.

\section{Energy Efficiency}

Researchers have used several approaches to address the problem of designing energy efficient IoT networks:

(a) developing energy-efficient routing protocols to reduce the number of hops (Machado et al., 2013), optimizing communication link status (Khan et al., 2016), adopting wakesleep strategies based on network traffic (Xia and Li, 2013), and data reduction via controlling the network topology.

(b) incorporating renewable energy devices in the network alongside adopting load-balancing strategies (Li et al., 2015; Han et al., 2016).

(c) exploiting wireless charging mechanisms to solve the fundamental issue of power management, especially for largescale heterogenous IoT networks (Madhja et al., 2016). However, from a hardware perspective there is a significant need to develop net-zero-energy sensor nodes, since the current trend is to pack an energy constrained node with more and more functionality, which could lead to a possible compromise between fidelity and power efficiency.

\section{GRAND CHALLENGES IN SENSING}

Sensing constitutes a vital part within IoT and wireless sensor networks. In a typical setting, sensed data is sent over the IoT network for post-analysis and inference to get insights. For the post-processing inference to be valuable, the accuracy of sensors is fundamental. For this accord, ISO standard 5725:1994 separates accuracy into precision and trueness while emphasizing the integrity of a sensor (Suzuki et al., 2019). In contrast, RF sensing utilizes the channel state information for sensing and, due to its inherent EM nature, it utilizes machine learning for classifying sensed information and presents additional challenges, such as linearity, repeatability, resolution, hysteresis, temperature coefficients, stability, and calibration.

Addressing these challenges consequently improves the reliability of RF sensing, ultimately realizing a possible future for ambient RF sensing. Furthermore, RF sensing has the advantage of a tactile nature, making it feasible for use in numerous applications, easily deployable, and cost-effective. The subsequent advances in ML techniques and RF characterization are envisaged to make RF sensing an integral part of IoT networks. This is also evident from the recent developments in the $\mathrm{THz}$ band for sensing and communications. Currently, $\mathrm{THz}$ is potentially being used for sensing food and water security (Ren et al., 2019a,b), sensing the cornea of eyes (Ozheredov et al., 2018), freezing of gait detection in Parkinson's disease (Tahir et al., 2019), sensing for real time near field imaging (Hillger et al., 2018), detecting corneal tissue water content (Grundfest et al., 2019), etc. Nevertheless, for the true success of IoT, we envisage that a variety of sensing instruments will be part of the network and the above challenges would need to be effectively addressed.

\section{VERTICALS OF IOT AND SENSOR NETWORKS}

\section{Road and Transportation}

By using wireless sensor networks (WSM) on roads, timely signaling control, precise traffic prediction, and road environment monitoring can be realized with high reliability (Pascale et al., 2012; Ramson and Moni, 2017). However, there are also some challenges associated with the design of WSNbased transportation systems. From the power consumption aspect, various solutions have been proposed to achieve balance between cost and reliability (Qin et al., 2010), such as cooperative transmission with reduced power consumption (Bai et al., 2008) or redesigning the duty cycle of the nodes (Lai et al., 2007; Nan et al., 2008). From the latency requirement aspect, various grant-based and grant-free-based access techniques are discussed (Birk et al., 2009; Franceschinis et al., 2009; Qin et al., 2010; Losilla et al., 2011). However, given the scarce resources and heterogeneous and dynamic characteristics of WSN in transportation systems, an efficient resource allocation scheme 
and network management are challenges to be dealt with. Additionally, WSNs may be used for safety related monitoring and control, thus posing very high requirements on both device and communication reliabilities.

\section{Buildings}

Future smart buildings (SB) will be prime examples of cyberphysical systems, where physical and virtual worlds connect and work together. SBs are the epitome of big data. However, there are several critical issues that hinders the adoption of this technology, such as security, user privacy, contextaware computing, and personal data stream management. To maintain the confidentiality and integrity of the SB data, various privacy-preserving encryption techniques have been proposed (Islam et al., 2012; Pérez et al., 2018). From a device perspective, there is a clear research trend of utilizing machine learning methods for anomaly detection and resolution in building management systems (Araya et al., 2016), however there is still a need to improve privacy enhancement technologies for user identity (Bandyopadhyay and Sen, 2011). Additionally, context-aware computing is an essential element of SB systems, however due to complex dependencies between humans and the apps, formalizing an accurate model is still a challenge for the current systems (Stankovic, 2014). Likewise, interconnecting multiple SB systems and processing large streams of heterogenous information are some of the open challenges that must be addressed through agreement on architectural and technological standards, as well as development of advanced and efficient algorithms.

\section{Healthcare}

It is evident that future healthcare systems will rely on an interplay of various technologies, including communication, sensing, cloud, and data analytics. From a communication perspective, short-range and long-range communication technologies are both needed to realize an end-to-end system. This leads to a co-existence issue between the two communication technologies, and therefore, SmartBAN or IEEE 802.15.6 has been proposed, which offers both in-body and off-body communication (Hämäläinen et al., 2015). However, the bottleneck is that the complaint devices are still not currently available in the market. On the other hand, the non-cellular and cellular low-power wide area network (LPWAN) technologies suffer from quality of service and high energy consumption issues, respectively (Alam et al., 2018). In terms of data analytics, designing of explainable AI algorithms is a real challenge and Complex Event Processing (CEP) domain is a promising research area to explore for designing future healthcare systems (Sandha et al., 2017). Lastly, data privacy is a key challenge for healthcare applications; sophisticated models of in-memory computation and processing and federated learning schemes are promising ways forward (Viceconti et al., 2015).

\section{Supply Chain-Farm to Folks}

A proactive supply chain, based on the IoT networking, has become increasingly essential for the growing population. An effective supply chain helps in attacking the problem well in time, along with reducing all potential threats and risks (Arora et al., 2020). Internet of things relying on sensors, radio frequency identification tags (RFID), and wireless communications can help in tracking and tracing products along the entire supply chain and in delivering the right product to the right place at the right time to the right person. Furthermore, movement and quality of the supply chain products can be monitored while streamlining the problematic association of goods. For IoT to complement the supply chain, it relies on good connectivity to be able to transmit their positions to GPS satellites or RFID receivers (Lee, 2020). It also relies on being installed and powered properly, demanding the use of trained personnel for handling, attaching, and removing. With a variety of sensors and devices, it is also crucial to choose the right device for the right job.

\section{Education and Training}

There exists a huge potential for education and training sectors to couple the collected information from IoT nodes with other data sources, including user mobility and data analytics, to enable new services and experiences (Abdelrahman et al., 2017). There are studies that capitalize on this collected information to understand user learning patterns, quantifying their online learning experience by measuring their concentration levels, measuring the impact of ease of accessibility of the learning content (Banica et al., 2017), and collecting IoT-based feedback. Likewise, the use of electronic devices was shown to be an effective pedagogical tool during a pilot study for students undertaking vocational training. However, there exists challenges in adopting IoT-based educational solutions, such as wireless coverage, high sensor costs, battery life, and crucially a lack of standard security and privacy measures. The adoption of IoTbased solutions for education is still very scarce, and there is room to further examine the factors that hinders the deployment of IoT solutions in the education sector (Al-Emran et al., 2020).

\section{Data Collection Storage and Processing}

As a massive number of sensors will be installed, data collection would mainly be concerned with the idea of how to implement it efficiently, where relative data compressing would be considered (Wu et al., 2014; Karim and Al-kahtani, 2016). To ensure the security of data collection, sensitive data needs to be protected by ciphering and leveraging the distributed system (Luo et al., 2018; Tao et al., 2019). Optimum energy transmission and path selection will also be considered to reduce the energy consumption (Orsino et al., 2016; Ang et al., 2018). Data storage, as the next step, is facing the same problems for energy reservation and data compressing, where some solutions are given through the improvement of data routing (Xu et al., 2015) and popular blockchain tech to secure data storage (Shafagh et al., 2017). As data processing might consider each corner of the whole IoT sensor network, the problems include, but are not limited to, in-network processing, data aggregation, and computing where relative signal processing techs are applied to solve the issues (Karim and Al-kahtani, 2016; Ahmed et al., 2017; Kim et al., 2017; Firoozi et al., 2018). 


\section{CONCLUSION AND OUTLOOK}

New technologies and applications are surfacing every day; however, there still exists challenges and gaps that need to be addressed. In this editorial article, we have outlined a few important challenges in Io $\mathrm{T}$ and sensing technologies and addressed important issues, including scalability, selforganization, security, and energy efficiency that must be catered for when deploying this emerging technology for the betterment of our society. Additionally, advancement in sensing technologies will pave the way for effective IoT solutions and, as outlined in this article, will fuel innovation across various verticals including transport, healthcare, buildings, supply chains, education, and many aspects of our daily lives. The progress toward the standardization of architectures, cross-sector application designs, device interoperability, privacy and end-to-end security, and highly efficient multi-standard communication systems and sensors, along with innovative device charging mechanisms, will inevitably bridge the gap between the physical and digital world

\section{REFERENCES}

Abdelrahman, A., Abusaimeh, H., Hammoudeh, M., Uliyan, D., Abu-Hashem, M. A., Murad, S., et al. (2017). "A survey on internet of things enabled smart campus applications," in Proceedings of the International Conference on Future Networks and Distributed Systems (ICFNDS '17) (New York, NY: Association for Computing Machinery), 1-7. doi: 10.1145/3102304. 3109810

Ahmed, E., Yaqoob, I., Hashem, I. A. T., Khan, I., Ahmed, A. I. A., Imran, M., et al. (2017). The role of big data analytics in internet of things. Comput. Netw. 129, 459-471. doi: 10.1016/j.comnet.2017.06.013

Alam, M. M., Malik, H., Khan, M. I., Pardy, T., Kuusik, A., and Le Moullec, Y. (2018). A Survey on the roles of communication technologies in IoTbased personalized healthcare applications. IEEE Access 6, 36611-36631. doi: 10.1109/ACCESS.2018.2853148

Al-Emran, M., Malik, S. I., and Al-Kabi, M. N. (2020). "A survey of internet of things (IoT) in education: opportunities and challenges," in Toward Social Internet of Things (SIoT): Enabling Technologies, Architectures and Applications. Studies in Computational Intelligence, Vol. 846, eds A. Hassanien, R. Bhatnagar, N. Khalifa, and M. Taha (Cham: Springer), 197-210. doi: 10.1007/978-3-030-24513-9_12

Ang, K. L., Seng, J. K. P., and Zungeru, A. M. (2018). Optimizing energy consumption for big data collection in largescale wireless sensor networks with mobile collectors. IEEE Syst. J. 12, 616-626. doi: 10.1109/JSYST.2016.26 30691

Araya, D. B., Grolinger, K., ElYamany, H. F., Capretz, M. A., and Bitsuamlak, G. (2016). "Collective contextual anomaly detection framework for smart buildings," in IEEE International Joint Conference on Neural Networks (IJCNN) (Vancouver, BC), 511-518. doi: 10.1109/IJCNN.2016.7727242

Arora, R., Haleem, A., and Arora, P. K. (2020). "Impact of IoT-enabled supply chain-a systematic literature review," in Proceedings of International Conference in Mechanical and Energy Technology. Smart Innovation, Systems and Technologies, Vol. 174, eds S. Yadav, D. Singh, P. Arora, and H. Kumar (Singapore: Springer), 513-518. doi: 10.1007/978-981-15-2647-3_46

Bai, L., Zhao, L., and Liao, Z. (2008). "Energy balance in cooperative wireless sensor network," in 14th IEEE European Wireless Conference (Prague), 1-5.

Bandyopadhyay, D., and Sen, J. (2011). Internet of things: applications and challenges in technology and standardization. Wireless Pers. Commun. 58, 49-69. doi: 10.1007/s11277-011-0288-5

Banica, L., Burtescu, E., and Enescu, F. (2017). The impact of internet-of-things in higher education. Sci. Bull. Econ. Sci. 16, 53-59. and create information societies and knowledge economies. The combination of tactile internet and ultra-responsive and ultra-reliable low-latency communication offered by $5 \mathrm{G}$ systems will add a new dimension to human-machine interaction and future IoT applications and will further push for the rise of fog and mobile edge computing architecture. There are many hyperconnected actors in this evolving ecosystem, whether that be machines, organizations, algorithms, or humans, and if we want the digital and physical world to go hand in hand, these relationships must be managed well through well-defined policies and regulations.

\section{AUTHOR CONTRIBUTIONS}

MI led the presented idea. MI, AZ, LZ, and QA contributed to the writing of the manuscript. AZ led the editing of the manuscript, and MI further provided the critical feedback and editing to help shape the manuscript. All authors contributed to the article and approved the submitted version.

Bi, S., Zhang, R., Ding, Z., and Cui, S. (2015). Wireless communications in the era of big data. IEEE Commun. Mag. 53, 190-199. doi: 10.1109/MCOM.2015.7295483

Birk, W., Osipov, E., and Eliasson, J. (2009). "iRoad-cooperative road infrastructure systems for driver support," in World Congress and Exhibition on Intelligent Transport Systems and Services: 21/09/2009-25/09/2009 (Stockholm: Curran Associates, Inc.).

Cabré, J. A. C., Precup, D., and Sanz, R. (2017). "Horizontal and vertical selfadaptive cloud controller with reward optimization for resource allocation," in 2017 International Conference on Cloud and Autonomic Computing (ICCAC) (Tucson, AZ: IEEE). doi: 10.1109/ICCAC.2017.25

Chen, S., Xu, H., Liu, D., Hu, B., and Wang, H. (2014). A vision of IoT: applications, challenges, and opportunities with china perspective. IEEE Internet Things J. 1, 349-359. doi: 10.1109/JIOT.2014.2337336

Cheng, B., Solmaz, G., Cirillo, F., Kovacs, E., Terasawa, K., and Kitazawa, A. (2017). Fogflow: easy programming of IoT services over cloud and edges for smart cities. IEEE Internet Things J. 5, 696-707. doi: 10.1109/JIOT.2017.2747214

Ding, Y., Jin, Y., Ren, L., and Hao, K. (2013). An intelligent self-organization scheme for the internet of things. IEEE Comput. Intell. Mag. 8, 41-53. doi: 10.1109/MCI.2013.2264251

Firoozi, F., Zadorozhny, V. I., and Li, F. Y. (2018). Subjective logicbased in-network data processing for trust management in collocated and distributed wireless sensor networks. IEEE Sens. J. 18, 6446-6460. doi: 10.1109/JSEN.2018.2848205

Franceschinis, M., Gioanola, L., Messere, M., Tomasi, R., Spirito, M. A., and Civera, P. (2009). "Wireless sensor networks for intelligent transportation systems," in VTC Spring 2009-IEEE 69th Vehicular Technology Conference (Barcelona: IEEE), 1-5. doi: 10.1109/VETECS.2009.5073915

Grundfest, W. S., Taylor, Z., Garritano, J., Nowroozi, B., Bajwa, N., and Sung, S. (2019). THz Sensing of Corneal Tissue Water Content. U.S. Patent Application 16/093953.

Hämäläinen, M., Paso, T., Mucchi, L., Girod-Genet, M., Farserotu, J., Tanaka, H., et al. (2015). "ETSI TC SmartBAN: overview of the wireless body area network standard," in Proceedings of 9th International Symposium on Medical Information and Communication Technology (ISMICT) (Kamakura), 1-5. doi: 10.1109/ISMICT.2015.7107485

Han, Q., Yang, B., Chen, C., and Guan, X. (2016). Energy-aware and QoS-aware load balancing for HetNets powered by renewable energy. Comput. Netw. 94, 250-262. doi: 10.1016/j.comnet.2015.10.025

Hillger, P., Jain, R., Grzyb, J., Mavarani, L., Heinemann, B., Mac Grogan, G., et al. (2018). “A 128-pixel $0.56 \mathrm{THz}$ sensing array for real-time near-field imaging 
in $0.13 \mu \mathrm{m}$ SiGe BiCMOS," in 2018 IEEE International Solid-State Circuits Conference-(ISSCC), 418-420. doi: 10.1109/ISSCC.2018.8310362

Islam, K., Shen, W., and Wang, X. (2012). "Security and privacy considerations for wireless sensor networks in smart home environments," in Proc. IEEE 16th International Conference on Computer Supported Cooperative Work in Design (CSCWD) (Wuhan), 626-633. doi: 10.1109/CSCWD.2012.6221884

Karim, L., and Al-kahtani, M. S. (2016). "Sensor data aggregation in a multilayer big data framework," in 2016 IEEE 7th Annual Information Technology, Electronics and Mobile Communication Conference (IEMCON) (Vancouver, BC), 1-7. doi: 10.1109/IEMCON.2016.7746261

Kephart, J. O. (2005). "Research challenges of autonomic computing," in Proceedings of 27th IEEE International Conference on Software Engineering, ICSE 2005 (Saint Louis, MO), 15-22. doi: 10.1145/1062455.10 62464

Khan, A. A., Rehmani, M. H., and Reisslein, M. (2016). Cognitive radio for smart grids: survey of architectures spectrum sensing mechanisms and networking protocols. IEEE Commun. Surv. Tuts. 18, 860-898. doi: 10.1109/COMST.2015.2481722

Kim, K., Min, S., and Han, Y. (2017). "A programmable data plane to support in-network data processing in software defined IoT," in 2017 International Conference on Information and Communication Technology Convergence (ICTC) (Jeju), 855-860. doi: 10.1109/ICTC.2017.8190798

Lai, Y., Zheng, Y., and Cao, J. (2007). "Protocols for traffic safety using wireless sensor network," in International Conference on Algorithms and Architectures for Parallel Processing (Berlin; Heidelberg: Springer), 37-48. doi: 10.1007/978-3-540-72905-1_4

Lee, I. (2020). “The Internet of Things (IoT): capabilities and applications for smart supply chain," in Securing the Internet of Things: Concepts, Methodologies, Tools, and Applications, ed Management Association, I (IGI Global), 1557-1574. doi: 10.4018/978-1-5225-9866-4.ch071

Li, M., Nishiyama, H., Kato, N., Owada, Y., and Hamaguchi, K. (2015). On the energy-efficient of throughput-based scheme using renewable energy for wireless mesh networks in disaster area. IEEE Trans. Emerg. Topics Comput. 3 , 420-431. doi: 10.1109/TETC.2014.2386135

Losilla, F., Garcia-Sanchez, A. J., Garcia-Sanchez, F., Garcia-Haro, J., and Haas, Z. J. (2011). A comprehensive approach to WSN-based ITS applications: a survey. Sensors 11, 10220-10265. doi: 10.3390/s111110220

Luo, E., Bhuiyan, M. Z. A., Wang, G., Rahman, M. A., Wu, J., and Atiquzzaman, M. (2018). Privacy protector: privacy protected patient data collection in IoT-based healthcare systems. IEEE Commun. Mag. 56, 163-168. doi: 10.1109/MCOM.2018.1700364

Machado, K., Rosario, D., Cerqueira, E., Loureiro, A. A., Neto, A., and de Souza, J. N. (2013). A routing protocol based on energy and link quality for internet of things applications. Sensors 13, 1942-1964. doi: 10.3390/s1302 01942

Madhja, A., Nikoletseas, S., and Raptis, T. P. (2016). Hierarchical collaborative wireless energy transfer in sensor networks with multiple mobile chargers. Comput. Netw. 97, 98-112. doi: 10.1016/j.comnet.2016. 01.007

Milner, S., Davis, C., Zhang, H., and Llorca, J. (2012). Nature-inspired selforganization control and optimization in heterogeneous wireless networks. IEEE Trans. Mobile Comput. 11, 1207-1222. doi: 10.1109/TMC.2011.141

Nan, D., Guozhen, T., Honglian, M., Mingwen, L., and Yao, S. (2008). "Low-power vehicle speed estimation algorithm based on WSN," in 2008 11th International IEEE Conference on Intelligent Transportation Systems (IEEE), 1015-1020. doi: 10.1109/ITSC.2008.47 32648

Orsino, A., Araniti, G., Militano, L., Alonso-Zarate, J., Molinaro, A., and Iera, A. (2016). Energy efficient iot data collection in smart cities exploiting D2D communications. Sensors 16:836. doi: 10.3390/s160 60836

Ozheredov, I., Prokopchuk, M., Mischenko, M., Safonova, T., Solyankin, P., Larichev, A., et al. (2018). In vivo $\mathrm{THz}$ sensing of the cornea of the eye. Laser Phys. Lett. 15:055601. doi: 10.1088/1612-202X/aaac76

Pang, J., Huang, Y., Xie, Z., Han, Q., and Cai, Z. (2020). Realizing the heterogeneity: a self-organized federated learning framework for IoT. IEEE Internet Things J. doi: 10.1109/JIOT.2020.30 07662
Pascale, A., Nicoli, M., Deflorio, F., Dalla Chiara, B., and Spagnolini, U. (2012). Wireless sensor networks for traffic management and road safety. IET Intell. Transport Syst. 6, 67-77. doi: 10.1049/iet-its.2010. 0129

Pérez, S., Hernández-Ramos, J. L., Matheu-García, S. N., Rotondi, D., Skarmeta, A. F., Straniero, L., et al. (2018). A lightweight and flexible encryption scheme to protect sensitive data in smart building scenarios. IEEE Access 6, 11738-11750. doi: 10.1109/ACCESS.2018.28 01383

Qin, H., Li, Z., Wang, Y., Lu, X., Zhang, W., and Wang, G. (2010). “An integrated network of roadside sensors and vehicles for driving safety: concept, design and experiments," in 2010 IEEE International Conference on Pervasive Computing and Communications (PerCom) (Mannheim: IEEE), 79-87. doi: 10.1109/PERCOM.2010.5466988

Qiu, T., Chen, N., Li, K., Atiquzzaman, M., and Zhao, W. (2018). How can heterogeneous internet of things build our future: a survey. IEEE Commun. Surv. Tutor. 20, 2011-2027. doi: 10.1109/COMST.2018.2803740

Qiu, T., Zhao, A., Xia, F., Si, W., and Wu, D. O. (2017). ROSE: robustness strategy for scale-free wireless sensor networks. IEEE/ACM Trans. Netw. 25, 2944-2959. doi: 10.1109/TNET.2017.2713530

Ramson, S. R. J., and Moni, D. J. (2017). "Applications of wireless sensor networks-a survey," in 2017 International Conference on Innovations in Electrical, Electronics, Instrumentation and Media Technology (ICEEIMT) (Coimbatore: IEEE), 325-329.

Ren, A., Zahid, A., Fan, D., Yang, X., Imran, M. A., Alomainy, A., et al. (2019a). State-of-the-art in terahertz sensing for food and water security-a comprehensive review. Trends Food Sci. Technol. 85, 241-251. doi: 10.1016/j.tifs.2019.01.019

Ren, A., Zahid, A., Zoha, A., Shah, S. A., Imran, M. A., Alomainy, A., et al. (2019b). Machine learning driven approach towards the quality assessment of fresh fruits using non-invasive sensing. IEEE Sens. J. 20, 2075-2083. doi: 10.1109/JSEN.2019.2949528

Sandha, S. S., Kachuee, M., and Darabi, S. (2017). Complex event processing of health data in real-time to predict heart failure risk and stress. arXiv 1707.04364.

Shafagh, H., Burkhalter, L., Hithnawi, A., and Duquennoy, S. (2017). "Towards blockchain-based auditable storage and sharing of IoT data," in Proceedings of the 2017 on Cloud Computing Security Workshop (New York, NY), 45-50. doi: $10.1145 / 3140649.3140656$

Shirer, M., and MacGillivray, C. (2020). The Growth in Connected IoT Devices is Expected to Generate 79.4ZB of Data in 2025. According to a New IDC Forecast. Available online at: https://www.idc.com/getdoc.jsp? containerId=prUS45213219 (Accessed 26 November, 2020).

Sicari, S., Rizzardi, A., Grieco, L. A., and Coen-Porisini, A. (2015). Security, privacy and trust in internet of things: the road ahead. Comput. Netw. 76, 146-164. doi: 10.1016/j.comnet.2014.11.008

Stankovic, J. A. (2014). Research directions for the internet of things. IEEE Internet Things J. 1, 3-9. doi: 10.1109/JIOT.2014.2312291

Sun, Y., Zhang, L., Feng, G., Yang, B., Cao, B., and Imran, M. (2019). "Performance analysis for blockchain driven wireless iot systems based on tempo-spatial model," in International Conference on Cyber-Enabled Distributed Computing and Knowledge Discovery (CyberC) (Guilin), 348-353. doi: 10.1109/CyberC.2019.00066

Suzuki, T., Takeshita, J. I., Ogawa, M., Lu, X. N., and Ojima, Y. (2019). "Analysis of measurement precision experiment with categorical variables," in 13th International Workshop on Intelligent Statistical Quality Control 2019, IWISQC 2019 (Hong Kong).

Tahir, A., Ahmad, J., Shah, S. A., Morison, G., Skelton, D. A., Larijani, H., et al. (2019). WiFreeze: multiresolution scalograms for freezing of gait detection in Parkinson's leveraging 5G spectrum with deep learning. Electronics 8:1433. doi: $10.3390 /$ electronics 8121433

Tao, H., Bhuiyan, M. Z. A., Abdalla, A. N., Hassan, M. M., Zain, J. M., and Hayajneh, T. (2019). Secured data collection with hardware-based ciphers for IoT-based healthcare. IEEE Internet Things J. 6, 410-420. doi: 10.1109/JIOT.2018.2854714

Viceconti, M., Hunter, P., and Hose, R. (2015). Big data big knowledge: big data for personalized healthcare. IEEE J. Biomed. Health Inform. 19, 1209-1215. doi: $10.1109 /$ JBHI.2015.2406883 
Wu, M., Tan, L., and Xiong, N. (2014). A structure fidelity approach for big data collection in wireless sensor networks. Sensors 15, 248-273. doi: $10.3390 / \mathrm{s} 150100248$

Xia, D. F., and Li, Q. (2013). "A routing protocol for congestion control in RFID wireless sensor networks based on stackelberg game with sleep mechanism," in IEEE 12th International Symposium on Distributed Computing and Applications to Business, Engineering \& Science (DCABES) (Los Alamitos, CA), 207-211. doi: 10.1109/DCABES.2013.44

Xu, H., Zhang, L., Liu, Y., and Cao, B. (2020). RAFT based wireless blockchain networks in the presence of malicious jamming. IEEE Wireless Commun. Lett. 9, 817-821. doi: 10.1109/LWC.2020.2971469

Xu, J., Guo, S., Xiao, B., and He, J. (2015). Energy-efficient big data storage and retrieval for wireless sensor networks with nonuniform node distribution. Concurr. Comput. Pract. Exp. 27:8. doi: 10.1002/cpe.3645
Xu, Y., and Helal, A. (2015). Scalable cloud-sensor architecture for the internet of things. IEEE Internet Things J. 3, 285-298. doi: 10.1109/JIOT.2015.2455555

Conflict of Interest: The authors declare that the research was conducted in the absence of any commercial or financial relationships that could be construed as a potential conflict of interest.

Copyright (c) 2020 Imran, Zoha, Zhang and Abbasi. This is an open-access article distributed under the terms of the Creative Commons Attribution License (CC BY).

The use, distribution or reproduction in other forums is permitted, provided the original author(s) and the copyright owner(s) are credited and that the original publication in this journal is cited, in accordance with accepted academic practice. No use, distribution or reproduction is permitted which does not comply with these terms. 